\title{
Zur Kenntniss der Acne frontalis seu varioliformis (Hebra), Acne frontalis necrotica (Boeck).
}

\author{
Von
}

\author{
Prof. F. J. Pick in Prag.
}

(Hiezu Tafel V.)

Es ist nicht leicht zu sagen, was die Autoren unter dem Namen Acne varioliformis verstehen. Wenn man auch von dem Umstande absieht, dass französische Dermatologen nach dem Vorgange Bazin's diese Bezeichnung noch immer für die nun allgemein als Molluscum contagiosum oder Epithelioma molluscum benannte Krankheit gebrauchen, so ist doch anderseits sicher, dass diese Benennung noch für ganz verschiedene Krankheitsprocesse angewendet werde. Schon die Erklärungen, welche für das Epitheton "varioliformis" gegeben werden, lassen die Verwirrung ahnen. Bald sollen die Efflorescenzen Aehnlichkeit mit den bei der Variola haben und dies durch den Namen ausgedrückt werden, bald wieder soll die Krankheit durch die Hinterlassung von Narben, wie nach Variola, den Beinamen verdienen, bald soll beides zusammen den Grund für die Bezeichnung abgegeben haben.

Es steht schlimm nach dieser Richtung mit der Belehrung, deren wir aus den Hand- und Schulbüchern theilhaftig werden. Nicht alle Verfasser sind so offen wie E. Veiel, der in Ziemssen's Haudbuch der Hautkrankheiten II. Th., S. 223, nach einigen kurzen Angaben, die er Hebra's Handbuch entnommen hat, schliesslich erklärt, er habe diese Kraukbeit nie geseben. Augenscheinlich befanden sich auch die meisten anderen Verfasser in 
derselben Lage, allein sie lassen uns doch im Zweifel darüber, ob die mancherlei einander widersprechenden Angaben von ibnen selbst ermittelt wurden, oder von anderwärts stammen und woher.

Es war deshalb sehr zeitgemäss, dass Prof. Boeck1) durch die Mittheilung eines sehr interessanten Falles die Discussion über diese "versäumte Krankheit“ eröffnete und zugleich zur Kenntniss derselben einen erfreulichen Beitrag lieferte. ${ }^{2}$ )

Die Mittheilungen ron Boeck erweitern jedoch die Charakteristik, welche Hebra ron seiner Acne varioliformis gegeben liat in vielen wesentlichen Punkten ganz bedentend, die Angaben beider weichen von einander derart ab, dass es Jedermann klar sein muss, dass es sich bei Boeck um eine andere Form der Krankheit handelt, als die, welche Hebra im Ange hatte.

Im Nachfolgenden werde ich zwei Fälle mittheilen, welche dieses Verhältniss klarstellen werden. Ich wähle dieselben aus einer grösseren Zahl von Fällen, weil sie jeder in seiner Art typisch sind für die eine oder die andere Form.

Fall 1. (Hiezu die Abbildung auf Tafel V.) Acne varioliformis Hebra.

D. F., 30 Jahre alt, Kaufmann, kommt in die klinische Ambulanz wegen eines Ausschlages im Gesichte, der wegen seiner Aehnlichlkeit mit Blattern seine Kundsehaft ängstigt, ihn deshalb verhindert im Laden añwesend zu sein und auf diese Weise materiell schädigt.

Die Krankheit soll vor einem halben Jahre plötzlich mit der Entwickelung kleiner rothbrauner Knötchen auf der Nase begonnen haben, denen nach einigen Tagen ähnliche Knötchen auf den Wangen und an der Stirne folgten. Von da an bis zum heutigen Tage dauert der Process ununterbrochen fort, indem sich immer neue Kuötchen bilden, während die älteren mit Hinterlassung von Narben zur Rü̈ckbildung gelangen.

1) Cäsar Boeck: „Ueber Acne frontalis seu necrotica." Dieses Arehiv 1889, Heft. I.

$\left.{ }^{2}\right)$ Ich hatte gerade eine Mittheilung für das Archiv über denselben Gegenstand vorbereitet und die meiner Arbeit beigegebene Tafel war schon ausgeführt, als mir der Artikel des Herrn Collegen Boeck zur Aufnahme in das Archiv zukam. Ich habe seiner Arbeit den Vortritt gelassen, weil ich in vielen wesentlichen Punkten zu denselben Resultaten gekommen war und nicht den Verdacht auf mich laden wollte, Hern Collegen Boeck die Prioritat sehmäleru zu wollen. 
Dic Eltern des Patienten leben und sind gesund. Die Mutter ist 68 Jahre alt, der Vater 70. Von sechs Geschwistern leben vier, eine Schwester starb im 2. Lebensjahre an Variola, eine zweite war skoliotisch, starb im 22. Lebensjahre an Erstickung aus ihm unbekannter Ursache, die übrigen stehen im Alter von 33, 35̈, 41 Jahren und sind gesund. Patient ist der jüngste von ihnen, litt vor seinem 16. Lebensjahre häufig an Furunculosis, in diesem Jahre überstand er Variola. Vom 20. Lebensjabre an leidet er an mässiger Seborrhoea capillitii, die zu theilweisem Haarausfall führte. Zum Militär kam er nicht wegen starker Varicositäten an den Unterestremitäten.

Vor etwa zehn Jahren litt er durch sechs Wochen an Gonorrhöe. Anderweitige venerische oder syphilitische Infectionen hatte er nicht. Dagegen leidet er seit seiner Jugend bäufig an Halsentzündungen, die fast regelmässig zweimal des Jahres auftreten und mit stärkerem Fieber einhergehen. Patient ist zum zweiten Male verheiratet, aus der ersten Ehe stammt ein Knabe, der zwar schwächlich, aber sonst gesund sein soll, in zweiter Ehe ist er mit einer Witwe, die selbst zwei Kinder hat, verheiratet, aus dieser Ehe stammt ein Kind, das jetat acht Monate alt und vollkommen gesund ist. Abortus hat niemals bei seinen Frauen stattgefunden, die erste Frau starb an Tuberculose.

In der Familie sind ausser Variola keinerlei Ausschläge vorgekommen, auch jetzt leidet weder Frau noch Kinder an einem ähnlichen Ausschlage wie er. Er ist kein Alkoholiker.

Status: Patient ist mittelgross, mässig kräftig gebaut, Mușculatur schwach, Panniculus gering. Die Haut des Stammes und der Unterextremitäten zeigt, ausser mehreren pigmentirten Nävis, mehreren Mollusca fibromatosa und einem kleinen Angiom, keinerlei pathologische Veränderungen.

Die Haut des Gesichtes, der Stirne, insbesondere an der Haargrenze, den Ohrmuscheln, aber auch im behaarten Theile des Kopfes und zwischen den Barthaaren, zeigt äusserst reichlich, stecknadelkopf- bis linsengrosse, rothbraune, das Niveau beträchtlich überragende Knötchen und Knoten, deren Oberfläche theils gleichmässig gefärbt und glatt ist, theils central eine trockene braune Borke trägt.

Diese Borken haften sehr fest an ihrer Unterlage, erscheinen unter das Niveau des Knötchens eingesunken und werden an der Peripherie von dem nicht vertrockneten Theile des Knötchens umsäumt. Nach Entfernung der Borken liegt ein Substanzverlust zu Tage, von dem aus man mit der Sonde unter die unterminirten Ränder der Hantinfiltration gelangt. Unter der rechten 
Augenbraue ist eine grössere Zahl der Knötchen zu einer fast zusammenhängenden Geschwulst von $2 \mathrm{Mm}$. Länge und $1 \mathrm{Mm}$. Breite vereinigt.

Nur an sehr wenigen Efflorescenzen sieht man ausnahmsweise. an der Spitze einen gelben Eitersaum die Borke umgeben und das Knötchen selbst, das in diesem Falle eine beträchtliche Grösse erreicht, mit einem rothen Entzündungshofe versehen, gewöhnlich sind die braunrothen Knötchen scharf umschrieben und heben sich ebenso scharf von der sie umgebenden und unverändert aussehenden Haut ab.

Zwischendurch, ganz besonders an der Haargrenze, findet man der Grösse der Knoten oder Knötchen entsprechende tiefe, theils noch braunroth gefärbte, theils schon seröse Narben.

Ausser den angeführten Knötchen findet man zwischen denselben ähnliche, scheinbar unter der Haut liegende hanfkorn- bis kleinerbsengrosse Knoten, über denen die Hautdecke unverändert oder nur livid roth erscheint. Solche Knoten finden sich vorwiegend an den Seitentheilen des Halses, an den Handrücken nnd Vorderarmen.

Ein Zusammenhang mit den Haarbälgen ist selbst am behaarten Theile des Gesichtes und am behaarten Kopfe nicht ermittelbar. Ebensowenig ist ein Comedo nachweisbar oder an den Knötchen der Ausfübrungsgang einer Talgdrüse angedeutet.

Drückt man mit einem Bergh'schen Comedonenquetscher, von der dem Umfang des Knötchens angepassten Grösse, dasselbe umgreifend auf die Umgebung; so ist man im Stande, das ganze Knötchen oder den Inbalt desselben herauszuquetschen. Derselbe erweist sich als ein Körper von gelblicher wachsartiger Beschaffenheit, der zurückbleibende Substanzverlust erscheint seicht, die Blutung ist gering.

Wie schon aus der Abbildung ersichtlich ist, beginnt der Process mit den kleinen, stecknadelkopfgrossen braunrothen; leicht elevirten Knötchen, welche sich scharf ron der Umgebung abheben und entweder als solche schon den Rückbildungsprocess eingehen oder sich laugsam und verhältnissmässig wenig vergrössern.

Nach kurzem Bestande, oft schon an den kleinsten Knötchen, sieht man die centrale Borkenbildung, durch welche eben das 
Centrum des Knötchens gegenüber dem Rande einsinkt und die Efflorescenz ein dellenförmiges genabeltes Aussehen erhält.

Die Borke bleibt gewöhnlich sehr lange liegen und fällt, wean sie nicht vorher mechanisch entfernt wurde, orst ab, wenn sich unter ihr die Vernarbung vollzogen hat. Die Vernarbung geschieht zumeist ohne Eiterung unterhalb des Schorfes oder es kommt um den Schorf herum zu eiteriger Entzündung, in welchem Falle auch die äussere Form der Efflorescenzen sich ändert, indem sie bedeutend grösser werden, die Delle einbüssen, sich zuspitzen und um den Schorf herum eine gelbe, durch die Haut durchscheinende eiterige Flüssigkeitsansammlung zeigen und gewöhnlich, namentlich bei den grösseren Pusteln, einen rothen Entzündungshof darbieten.

Diese Entzündung greift viel tiefer als die Knoten reichen und hinterlässt dementsprechend anch viel tiefere Narben.

Durch innerliche Behandlung liess sich der Process in keinerlei Weise beeinflussen. Patient hatte, ehe er an die Klinik kam, durch viele Monate Jodkali und Solut. Fowleri genommen ohne die geringste Wirkung.

Ich habe die Knoten mit Bergh's Comedonenquetscher ausgedrückt oder mit dem scharfen Löffel entfernt, wodurch ich die vorhandenen Krankheitsherde am raschesten beseitigte.

Fall 2. Acne necrotica, Boeck. Der Fall betrifft den einundzwanzigjährigen, ledigen Knecht F. K., aufgenommen am 6. April 1889, sub Nr. 4337. Der Vater des Patienten leidet seit $z$ wei Jahren an einem arthritischen Processe, die Mutter und zwei Geschwister sind gesund, eine Schwester leidet an Epilepsie, vier Geschwister starben in früher Jugend. Patient selbst ist nie ernstlich krank gewesen, doch leidet er an seinem Ausschlage der im fünften Lebensjahre begann, in den folgenden Jahren periodisch wiederkehrte, um in den letzten Jahren bäufiger und unregelmässiger aufzutreten. Die Angaben des Patienten ïber das Auftreten des Ausschlages sind ganz genaue. Er sagt:

Vom fünften bis zum neunzehnten Lebensjahre trat der Ansschlag alljälurlich im Monate April im Gesichte auf und heilte nach vierzehntägigem Bestande vollkommen ab, während dieser ganzen Zeit war der Ausschlag mit keinerlei Beschwerden verbunden.

Im Jahre 1887 stellte sich der Ausschlag schon im Monate März ein, heilte in vierzehn Tagen ab, trat aber im April zweimıl auf, sobald Patient wieder zur Arbeit ging. Dar Ausschlag war diesmal mit Röthung und Schmerz in den Augen verbunden. 
Im Jahre 1889 erscheint das Exanthem schon Ende Januar, zugleich besteht eine starke Augenentzündung, sobald Patient wieder zar Arbeit geht, kehrt der Ausschlag nach wenigen Tagen im Monate Februar, März und April je zweimal wieder; Anfangs März war er mit einer so heftigen Augenentzundung verbunden, dass Patient nicht allein gehen konnte, damals liess sich Patient in das $\mathrm{H}$... . Spital und später auf die dermatologische Abtheilung aufnehmen. Der letzte Ausschlag im Monate April heilt nicht, wie die früheren mach vierzehntägigem Bestande aus, sondern besteht bis zum Herbste.

Im Jahre 1889 tritt das Exanthem am 20. Januar auf der linken Wange auf, besteht acht Tage, zugleich sind die Augen stark geröthet, am 14. Februar erscheint es wieder und dauert zwolf Tage, Ende Februar durch acht Tage hindurch.

Status vom 6. April: Körper klein, schwächlich gebaut, schlecht genährt, Musculatur wenig entwickelt, der Panniculus spärlich, die Hautfarbe blassbräunlich, das Haupthaar blond, sehütter, die Haut des behaarten Kopfes, besonders in der Stirnund Scheitelgegend mit sehr reichlichen trockenen, kleienartigen Schuppen bedeckt, die Haut im Allgemeinen schlecht gepflegt, gut eingeölt und normal durchfeuchtet.

Im Gesichte und am Stamm wenig, an den unteren Extremitäten sehr stark behaart, die extremen Körpertheile livid roth verfärbt. Hände und Füsse kühl anzufühlen, in den Hohltächen abnorm feucht.

Vollkommen symmetrisch localisirt, im ganzen Gesichte mit Ausnatime der Stirn, Lippen und Lider, besteht ein eigenartigés Exanthem. Dasselbe stellt sich der Hauptsache nach dar als circa linsengrosse, trockene, braunrothe Schorfe, die anf einem seichten Substanzverluste an der Spitze einer wenig elevirten und infiltrirten Partie ziemlich fest aufsitzen. Am Kinn und in der Regio parotidea beiderseits confluiren diese Schorfe zu grösseren Borken, auch kommt es daselbst aus dem stark gerötbeten, infiltrirten und erodirten Corium zu einem Ergusse seröser Flüssigkeit an die Oberfläche, wo dieselbe zu blätterartig an einander geschichteten Krusten eintrocknet. Die Anfangsstadien dieses Processes findet man augenscheinlich an beiden Ohrmuscheln und am oberen Rande der erkrạnkten Partien nach aussen vom linken äusseren Angenwinkel, und zwar stellen sich dieselben dar als gruppenweise zusammengedrängte, durch furchenartige Ein- 
Scbnürungen von einander getrennte knötchenförmige Erhebungen von 4-5 Mm. an der Basis, dieselben besitzen einen ziemlich lebhaften Glanz, sind blassroth und gelblich durchscheinend. In der Tiefe des einzelnen Herdes sieht man meist mehrere violette Punkte und Streifen durchscheinen. Eine Localisation an die Talgfollikel ist in diesem Stadium nicht bemerkbar, insoferne die einzelnen Erhebungen über eine grosse Zahl von Follikelöffungen ansgedehnt sind. Als Uebergangsstufe zwischen den beiden beschriebenen Formen des Exanthems bemerkt man nun einzelne solche Erhöhungen, die keine so pralle Spannung mehr darbieten, vielmehr abgeflacht sind, matt aussehen, zum Theil noch die erwähnten violetten Punkte durchschimmern lassen, oder schon einen opaken, bräunlichen, dünnen Schorf an der Spitze besitzen. Die Haut der Stirn und der Nase zeigt einen starken fettigen Glanz, ist fettig anzufühlen, im Bereiche der Nasenspitze und Flügel etwas geröthet, die Follikelöffnungen erweitert, mit Talgpfröpfen verstopft, die Drüsenkörper der Talgdrüsen schimmern durch die dünne Haut als hirsekorngrosse weisse Körner hindurch.

Im Harne keine abnormen Bestandtheile, überhaupt am übrigen Körper nichts Pathologisches.

13. April. Die Schorfe luaben sich sämmtlich abgelöst, unter denselben flache Narben. Die frischen Efforescenzen in der linken Schläfegegend haben sich derart verändert, dass sie allmälig weniger prall erscheinen, etwas opak sich färbten, statt der violetten Punlite im Innern eine gleichmässig braune Färbung annahmen und oberfächlich zu einem Schorfe eintrockneten.

21. April. Patient ging gesteru aus dem Zimmer. Hente unter Jucken frische Efflorescenzel am Kinn und in der Gegend der unteren Augenlider anfgetreten.

22. April. Gestern Spaziergang. Hente an den Wangen frische Efflorescenzen aufgetreten.

24. April. Annähernd in derselben Ausdehnung wie bei seinem Spitaleintritte erscheint nun die Haut des Gesichtes besetzt mit zahlreichen, oft dicht gedrängten, blassgelblichen Erhebungen von durchscheinender Beschaffenheit, welche den obersten Schichten angehören und in ibrer Tiefo zum Theile wenigstens jene violetten Punkte und Streifen orkennen lassen und, wie be- 
sonders am behaarton Theile der Oberlippe wahrnehmbar ist, sicher nicht ansschliesslich-an die Haarbälge gebunden sind. Bei seitlichem Druck wird schliesslich die Epidermisdecke durch ausgepresste seröse Flüssigkeit in Blasenform abgeboben und zum Platzen gebracht, beim seitlichen Anstechen der einzelnen Erhebungen entleert sich, gleichfalls erst durch seitlichen Druck, etwas Flüssigkeit. Entfernt man nach Aufhören des Druckes die Epitheldecke, so kommt ein blutig imbibirtes Corium zum Vorschein.

25. April. Das Exanthem, besonders im Bereich der linken Jochbein- und Wangengegend hat zum grössten Theile, in Folge nachfolgender Blntungen, eine mehr gleichmässige violette oder braune Färbung erhalten.

26. April. Die Efflorescenzen sind fast vollständig zu Krusten oder Schorfen eingetrocknet.

27. April. Sämmtliche Efflorescenzen eingetrocknet, die Haut an der Kinnlippenfurche, Nase, den Ohrmuscheln, Wangen mit mehr weniger umfänglichen Krusten und Schorfen bedeckt.

7. Mai. Das letzte Exanthem ist vollständig abgeheilt, neuerdings mit Hinterlassung zarter, wenig vertiefter, glatter weisser Narben. Gestern und heute schon hielt sich Patient eine Zeit lang im Freien auf. Heute Nachmittags erscheint die Haut des Gesichtes im Bereiche der Nase, der Wangen, der Ohrmuscheln und der Kinnfurche geröthet, glänzend, an der Nase und den Nasolabialfalten, sowie am Kinn stark fettig anzufühlen, die Temperatur derselben erhöht. Im Bereiche der linken Wange sieht und tastet man mehrere stumpfkegelförmige, annähernd hanfkorngrosse Frhöhungen von etwas durehscheinender Beschaffenheit.

9. Mai. Die Zahl der niedrigen, derben, gelblich durchscheinenden Erhebungen hat im Bereiche der beiden Wangen zugenommen, ist aber noch immer relativ gering. In der Kinnfurche und an den Ohrmuscheln sind einzelne neue aufgetreten, an mehreren zeigen sich bereits die eigenthümlichen, punktförmigen Blutaustritte, die besonders nach dem Abstreifen der blas enartigen Decke mit grosser Dentlichkeit hervortreten.

10. Mai. Hente sind keine neuen Eruptionen aufgetreten.

19. Mai. Der Kranke klagt über Schmerzhaftigkeit und 
Sehstörung am rechten Auge. Es zeigt sich auf der rechten Cornea eine centrale, punktförmige, graue Trübung. Leichte pericorneale Injection.

24. Mai. Viel stärkere pericorneale Injection.

29. Mai. An beiden Ohrmuscheln sind mehrere von Anfang an violette oder braun gefärbte Efflorescenzen aufgetreten.

Von nun an wurde der Kranke wieder im Zimmer zurückgehalten, es kamen weiter keine frischen Efflorescenzen zur Entwickelung und unter indifferentem Salbenverbande kam der Process wieder zu vollständiger Abheilung. Nachdem die Borken abgefallen waren, wurde ein Gelatinverband verordnet, seither sind bis zur Entlassung des Kranken, 26. Juni, keine frischen Nachschübe aufgetreten.

Es unterligt gar keinem Zweifel, dass es sich im ersten Falle um eine Acue varioliformis seu frontalis im Sinne Hebra's handelt. Seine Beschreibung von dieser Krankheit stimmt, ausser seinen Angaben von der ausschliesslichen Localisation „im Gesichte und auch da nur an der Stirne, hauptsächlich zunächst der behaarten Kopfhaut, sowie auch an letzterer hie und da zerstreut" vollständig mit unserem Bilde überein. Nach Hebra äussert sich diese Form der Acne theils durch mehr flache, hanfkorngrosse Knötchen, theils durch Pustelbildung, beherbergt im Innern keinen Comedo, bekommt an der Spitze ein flaches, scheibenförmiges Krustchen, welches den untergelagerten I'heilen fest adbärirt und später unter das Niveau des übrigen Knötchens gelangt, so zwar, dass der Rest des letzteren das centrale scheibeuförmige Börkchen allenthalben in Gestalt eines erhabenen Randes umgibt und hinterlässt, wenn die Borke abfällt eine Narbe, die gegen die Umgebung vertieft ist.

Abweichend von diesen Angaben ist, wie schon bemerkt, die Ausbreitung des Processes im Gesichte auf die Wangen und den behaarten Theil desselben, sowie auf die Ohrmuscheln, den Hals und die oberen Extremitäten.

Wie ich gezeigt habe, jst die Pustelbildung eine nur secundäre, offenbar nur durch dis Infection mit Fitercoccen an einzelnen Stellen um die verschorften Partien eingetretene Erscheinung. 
Wie die mikroskopische Untersuchung der ansgedrückten Knoten erweist, anderes Material ist mir nicht zur Verfügung gestanden, handelt es sich un Noubildung von Epithel, welches alsbald einer eigenartigen Degeneration verfällt, verschorft, während sich, wenn diese Neubildung in die Tiefe gegriffen, in der Umgebung der epithelialen Neubildung vom Chorion aus eine kleinzellige Infiltration entwickelt und zur Narbenbildung führt. Es ist mir nicht gelungen, Beziehungen zwischen den nengebildeten Epitbelknoten und den Talg- oder Haarbälgen nachzuweisen. Auch über die Degenerationsvorgänge an den neugebildeten Epithelien bin ich bisher nicht in der Lage otwas Bestimmtes auszusagen.

Immerhin bin ich der Ansicht, für diesen Process den Namen Acne varioliformis vorerst beizubehalten, weil er für das klinische Bild äusserst charakteristisch ist.

Ebenso unzweifelhaft ist es, dass es sich im zweiten Falle um jenen Process handelt, der mit dem in Boeck's Fallo identisch ist, und dem er im Gegensatze zu Hebra's Acneform den sehr zweckmässigen Namen Acne necrotica gegeben hat.

Ich acceptire den Namen für diese Krankheitsform, aber auch nur für diese, mit Vergnügen.

Sio charakterisirt sich ganz besonders durch die von Boeck treffend hervorgehobenen kleinen, staubförmigen, bämorrbagischen Pünktchen, die zusammen einen kleinen violettrothen Fleck bilden, ferner durch die ödematös imbibirte Oberhaut und die stellenweise viel stärkere und tiefer gehende Verschorfung.

Sie mit der Acne varioliformis Hebra zu identificiren, bin ich nicht in der Lage.

Inwiefern diese beidèn Krankheitsformen nahe verwandte oder zusammengehörige Processe darbieten, werden erst weitere Beobachtungen lehren.

Erklärung der Tafel im Texte. 


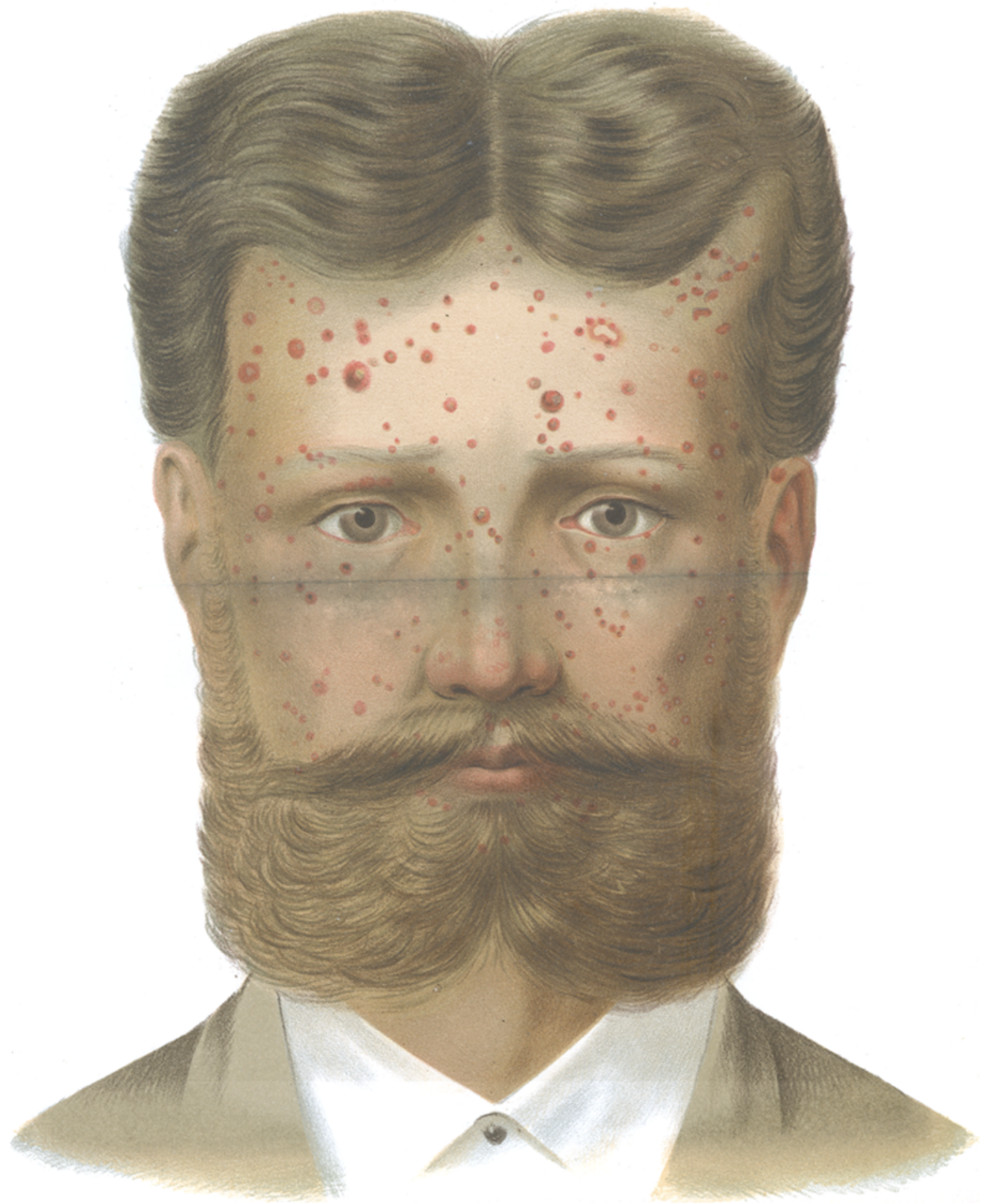

